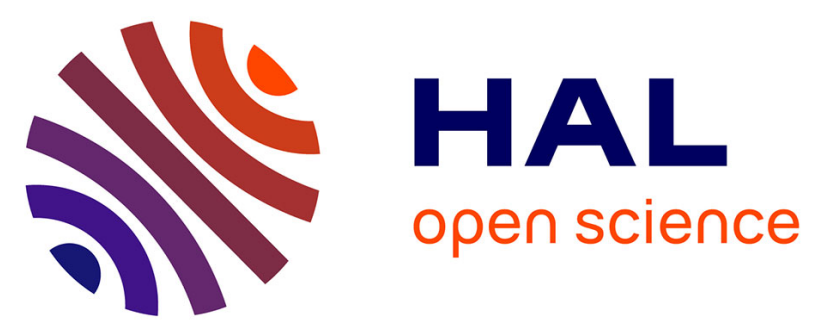

\title{
ETUDE DE L'ÉVOLUTION STRUCTURALE D'UN VERRE MÉTALLIQUE Fe83B14Si1,5C1,5 EN RELATION AVEC L'EFFET MAGNÉTOÉLASTIQUE ET L'AMORTISSEMENT MAGNÉTOMÉCANIQUE
}

\author{
F. Guyot, F. Fouquet, J. Perez
}

\section{To cite this version:}

F. Guyot, F. Fouquet, J. Perez. ETUDE DE L'ÉVOLUTIOn STRUCTURALE D'UN VERRE MÉTALLIQUE Fe83B14Si1,5C1,5 EN RELATION AVEC L'EFFET MAGNÉTOÉLASTIQUE ET L'AMORTISSEMENT MAGNÉTOMÉCANIQUE. Journal de Physique Colloques, 1983, 44 (C9), pp.C9-139-C9-144. 10.1051/jphyscol:1983916 . jpa-00223362

HAL Id: jpa-00223362 https://hal.science/jpa-00223362

Submitted on 1 Jan 1983

HAL is a multi-disciplinary open access archive for the deposit and dissemination of scientific research documents, whether they are published or not. The documents may come from teaching and research institutions in France or abroad, or from public or private research centers.
L'archive ouverte pluridisciplinaire HAL, est destinée au dépôt et à la diffusion de documents scientifiques de niveau recherche, publiés ou non, émanant des établissements d'enseignement et de recherche français ou étrangers, des laboratoires publics ou privés. 


\section{ETUde de L'ÉVOLUtion StRucturale d'un VERRE MÉtallique $\mathrm{Fe}_{83} \mathrm{~B}_{14} \mathrm{Si}_{1,5} \mathrm{C}_{1,5}$ EN RELATION AVEC L'EFFET MAGNÉTOÉLASTIQUE ET L'AMORTISSEMÉNT MÁGNÉTOMÉCANIQUE}

F. Guyot, F. Fouquet et J. Perez

Groupe d'Etudes de Métallurgie Physique et de Physique des Matériaux (LA 341), INSA de Lyon, Bat. 502, 20, Avenue A. Einstein, 69621 Vilzeurbanne Cedex, France

Résumé - Les variations de l'effet $\Delta E$ et de l'amortissement magnêtomécanique associées à l'évolution structurale d'un verre métallique ferromagnétique Fe$\mathrm{B}-\mathrm{Si}-\mathrm{C}$ ont êté étudiẻes. L'effet $\Delta E$ négligeable dans 1 'état brut d'hypertrempe devient très important après relaxation structurale de l'alliage puis disparait dès l'amorce de la cristallisation.

Abstract - The $\Delta E$ effect and magnetomechanical damping variations associated with the structural evolution of a Fe-B-Si-C ferromagnetic metallic glass have been studied. The $\Delta E$ effect negligible in the as-quenched state becomes very important after structural relaxation and then disappears from the onset of the crystallization.

\section{I - INTRODUCTION}

Des mesures de pouvoir thermoélectrique ainsi que des expériences de diffraction et de diffusion aux petits angles des rayons $X$ nous avaient permis de mettre en évidence les différentes étapes de 1 'évolution structurale d'un verre Fe83 $\mathrm{B}_{14} \mathrm{Si}_{1,5} \mathrm{C}_{1,5}$ hypertrempe depuis l'état liquide /1/: (i) relaxation structurale en phase vitreuse avec distinction de deux processus (mise en ordre topologique puis mise en ordre chimique avec ségrégation des mëtalioîdes) ; (ii) cristali isation primaire de fer a; (iji) devitrification de la phase amorphe restante avec cristallisation concomitante de la phase stable de type Fe 2 B et des phases métastables de type 0 - Fe3 B et $t$ - Fe3 $\mathrm{B}$ qui tendent à se décomposer par la suite en $\mathrm{Fe}-\alpha+\mathrm{Fe}_{2} \mathrm{~B}$.

Les techniques de mesure de moduie d'Young et de frottement intêrieur n'ont été, à ce jour, que très peu utilisées pour 1 'étude des verres métalliques $/ 2$ - $6 /$; elles sont pourtant susceptibles d'apporter des informations au niveau de la relaxation structurale notamment pour les verres ferromagnétiques par l'intermédiaire du couplage magnétoêlastique.

Notre objectif au cours de ce travail a donc été d'étudier les variations du module ẻlastique et du frottement intérieur du verre métallique ferromagnétique Fe83 $B_{14}$ $\mathrm{Si}_{1,5} \mathrm{C}_{1,5}$ en Tiaison avec nos résultats antérieurs sur 1 'évolution structurale de ce verre $/ 1,7 /$.

\section{II - METHODE EXPERIMENTALE}

Les mesures de module d'Young et de frottement intërieur on été réalisées par la méthode de la lame vibrante, l'échantillon étant encastré à une extrëmité et libre à l'autre. L'excitation et la détection du mouvement se font par voie électrostatique. Le système fonctionnant en boucle fermée, l'éprouvette vibre sur un mode propre avec une amplitude maintenue constante $\left(\simeq 2.10^{-6}\right)$. Le frottement intérieur est obtenu à partir du dēcrément logarithmique mesuré en décroissance libre après coupure de 1'excitation. Les dimensions des êchantilions sont $0,03 \times 1,1 \times 12$ mm3, la fréquence de vibration est alors de $150 \mathrm{~Hz}$ pour le mode fondamental et de $800 \mathrm{~Hz}$ pour le premier harmonique pour un échantillon brut d'hypertrempe. Les mesures sont rêalisables entre -196 et $+430^{\circ} \mathrm{C}$ sous un vide de $10-3$ Torr. 

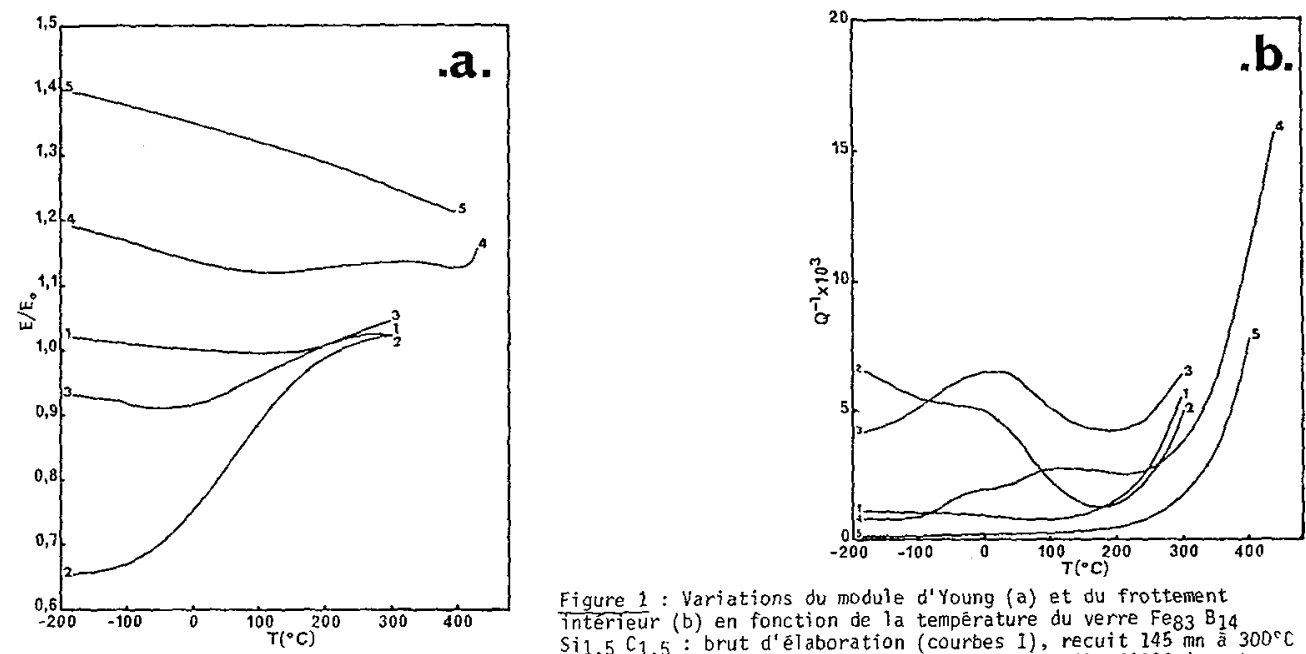

Figure 1 : Variations du module d'Young (a) et du frottement intérieur (b) en fonction de la température du verre $\mathrm{Fe}_{83} \mathrm{~B}_{14}$ Si $1,5 C_{1}, 5$ : brut d'élaboration (courbes 1), recuit 145 mn $300^{\circ} \mathrm{C}$ (courbes 2 ), après chauffage 辛 $400^{\circ} \mathrm{C}$ (courbes 3 ), $410^{\circ} \mathrm{C}$ (courbes 4) et $420^{\circ} \mathrm{C}$ (courbes 5 ) - $E_{0}$ valeur du module à $20^{\circ} \mathrm{C}$ pour $\uparrow$ 'échantillon brut d'hypertrempe.

\section{III - VARIATIONS OU FROTTEMENT INTERIEUR ET DU MODULE D' YOUNG EN FONCTION DE LA TEMPERATURE POUR DIFFERENTS ETATS STRUCTURAUX}

Les résultats sont représentés par les courbes des figures la et 1b. L'échantillon brut d'hypertrempe (courbes 1) présente une faible diminution du module d'Young et du frottement intérieur quand la tempërature augmente depuis $-196^{\circ} \mathrm{C}$ jusqu' à $100^{\circ} \mathrm{C}$; on peut donc penser qu'un effet magnéto-êlastique existe, son amplitude restant toutefois faible.

Les courbes 2 correspondent à un échantillon relaxé structuralement à $300^{\circ} \mathrm{C}$ mais toujours dans un état parfaitement vitreux. On constate alors par rapport aux courbes I une diminution de $E$ de l'ordre de $35 \%$ à $-196^{\circ} \mathrm{C}$, le module augmentant en outre fortement et le frottement intérieur diminuant (jusqu'à $200^{\circ} \mathrm{C}$ ) quand la température augmente. Ce comportement traduit l'existence d'un effet magnêto-êlastique $\triangle E$ et $d^{2}$ un amortissement magnéto-mécanique d'autant plus importants que la temparature est basse.

Les courbes 3 se rapportent à un échantillon dans lequel la cristallisation primaire de fer $\alpha$ a été faiblement amorcée. On observe alors que le module présente un minimum et le frottement intērieur un maximum aux environs de $0^{\circ} \mathrm{C}$, la valeur de $\mathrm{E}$ a $-196^{\circ} \mathrm{C}$ ayant fortement augmentée par rapport au cas précédent.

Les courbes 4 et 5 correspondent à une cristallisation primaire de fer $\alpha$ croissante mais n'affectant globalement qu'un faible volume de l'échantillon si l'on s'en réfère à $1 \mathrm{a}$ diffraction $\mathrm{X}$. On constate une augmentation progressive et importante du module, la valeur de $\mathrm{E}$ a $-196^{\circ} \mathrm{C}$ atteignant pour la courbe 5 le double de celle mesurée pour 1 a courbe 2 ; parallèlement le frottement intérieur diminue. Le minimum de module et le maximum de frottement intérieur observés sur les courbes 3, apparaissent encore sur les courbes 4 mais beaucoup moins marqués et décalés vers les hautes températures; ils disparaissent par contre complètement sur les courbes 5 .

Il est à noter que si l'on poursuit les traitements thermiques jusqu'à dévitrification totale de l'alliage le module d'Young $n^{\prime}$ augmente que de quelques pourcents supplémentaires ce qui parait indiquer qui une cristallisation très partielle du verre est suffisante pour supprimer à la fois l'effet magnēto-êlastique et l'anomalie de module entre états vitreux et cristallin. 


\section{IV - INTERPRETATION DES RESULTATS ET ANALYSE DES EFFETS MAGNETO-ELASTIQUES}

Nous avons pu constater (figure 1 courbes 2) qu'un recuit à $300^{\circ} \mathrm{C}$ du verre métallique ètudiē entraînait une chute importante du module d'Young que nous avons attribuē à un effet magnéto-élastique (effet $\Delta E$ ) ; une mesure du module à la température ambiante en plaçant 1'échantillon dans le champ d'un aimant permanent nous a conduit à une valeur de $E$ sensiblement ëgale à celle de l'échantillon.brut confirmant ainsi $l$ 'hypothëse de l'effet $\Delta E$.

Dans un verre métallique ferromagnétique, en 1'absence d'anisotropie magnétocristalline, le seul obstacle au couplage magnéto-élastique est constitué par les contraintes internes. Ces contraintes sont importantes dans $l^{\prime}$ 'état brut d'hypertrempe et $l^{\prime}$ 'effet $\Delta E$ est négligeable (courbes 1 figure 1 ). Un recuit à $300^{\circ} \mathrm{C}$ provoque une relaxation structurale et un relachement des contraintes internes d'oú 7 'apparition d'un effet $\Delta E$.

$L$ 'augmentation continue de cet effet $\Delta E$ et de l'amortissement magnétomécanique qui 1 'accompagne lorsqu' on descend en température jusqu'à $-196^{\circ} \mathrm{C}$, semble être une caractéristique distinguant les verres métalliques des matériaux cristallins ferromagnétiques tel le nickel. Berry et Pritchet /8/ ont en effet observé la même dépendance en température sur deux verres métalliques Fe-P-C et Fe-Ni-P-B recuits. Cette augmentation continue de l'effet $\Delta E$ ne peut être que la conséquence directe de 1 'absence d'anisotropie magnétocristalline et de 1 'augmentation de la magnétostriction à saturation lorsque la température décroit. Ceci est en accord avec les études de O'Handley et al /9/ sur la magnétostriction des verres Fe-B. L'apparition de cristaux de fer $\alpha$ au sein de la matrice amorphe jouant le rôte d'ancrages et de centres de contraintes va limiter le couplage magnétoêlastique. Le maximum de frottement intërieur observē (courbe 3 fig. 1b) peut alors s'expliquer par un dësancrage thermiquement active des parois de Bloch. Lorsque le nombre et la taille des cristaux de fer $\alpha$ sont suffisants le désancrage devient impossible et l'effet $\Delta E$ disparait.

Comme pour les matériaux cristallins, 1 'effet $\Delta E$ et 1 'amortissement magnétomécanique d'un verre métallique ferromagnétique peuvent avoir trois origines $/ 10 /$ :

- relaxation par courants de Foucault macroscopiques

- relaxation par courants de Foucault microscopiques

- hys têrésis magnétomécanique.

Dans l'état désaimanté ou l'êtat saturé la composante relaxationnelle macroscopique est nulle. Donc, dans notre cas n'interviennent que les deux autres contributions. En outre les processus de relaxation sont indëpendants de l'amplitude de 1a déformation $\varepsilon$ et dépendent de la fréquence $f$ tandis que la composante hystérétique est indëpendante de $f$ et dépend fortement de $\varepsilon$ s'annulant pour $\varepsilon=0$.

La figure 2 montre les variations de $E$ et que $Q^{-1}$ en fonction de la déformation $\varepsilon_{\max }$. pour deux fréquences différentes $130 \mathrm{~Hz}$ (2a) et $800 \mathrm{~Hz}$ (2b). On constate que $E$ et $Q^{-1}$ varient lineairement avec $\varepsilon_{\max }$. pour $2.10^{-6}<\varepsilon_{\max }<10^{-5}$ et qu'à plus basse amplitude ils tendent asymptotiquement vers une limite qui doit correspondre à 1 a suppression de tout phénomène hystérétique. Nos résultats (fig. 1) ayant été obtenus pour $\varepsilon_{\max .} \simeq 2 \cdot 10^{-6}$ la contribution hystérétique ne peut qu'être faibie.

La valeur du frottement intérieur mesurē à très faible déformation devrait donc correspondre au processus de relaxation par courants de Foucault microscopiques qui peut être décrit par un spectre de relaxation $\left(g_{k}, v_{k}\right),(k=1,2,3 \ldots) / 10 \%$.

La première fréquence du spectre étant égale à : $\nu_{1}=\frac{10^{9} \rho}{2 \mu_{r} d^{2}}$ avec d dimension

moyenne des domaines (en $\mathrm{cm}$ ), $\rho$ rêsistivité êlectrique $(\Omega . \mathrm{cm})$, $\mu_{r}$ perméabilité réversible ( $\mu_{r}=\mu_{0}$ dans l'état désaimantê).

A partir des données expérimentales de la littérature concernant les alliages 

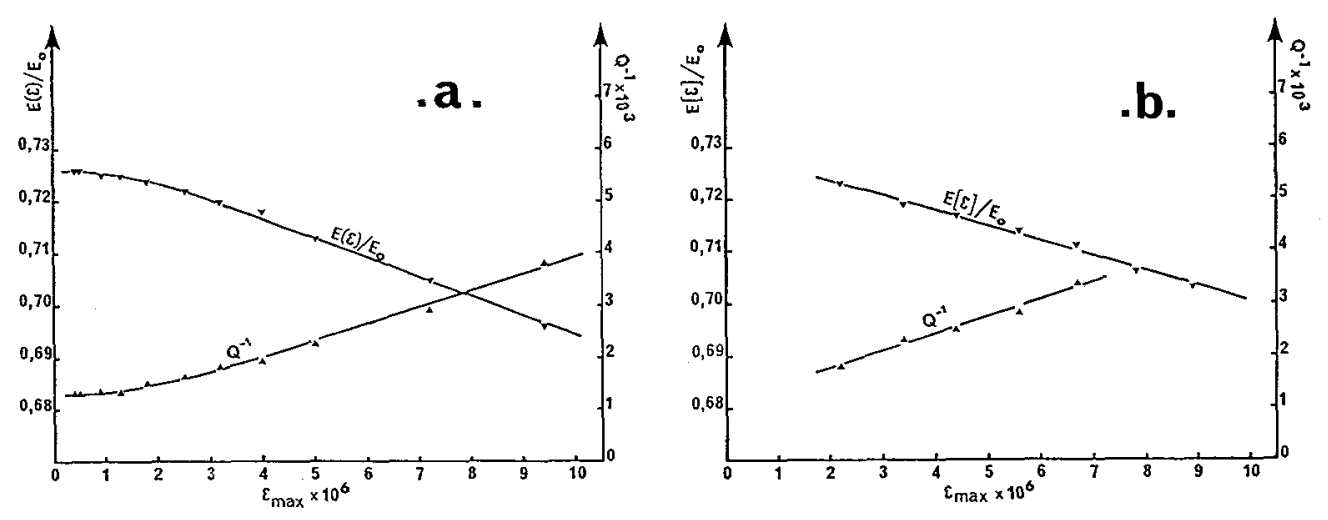

Figure 2 : Variation du module d'Young et du frottement intérieur en fonction de

$\varepsilon_{\max }$ pour un échantillon recuit a $300^{\circ} \mathrm{C}:(\mathrm{a})$ mesure réalisée sur le mode fondamen-

tal de vibration (b) mesure réalisée sur ie premier harmonique.

Fe-B $/ 11 /$ on obtient $\nu_{1}=20 \mathrm{khz}$ pour une taille moyenne de domaine $d=300 \mu \mathrm{m}$ et $\nu_{1}=2 \mathrm{MHz}$ pour $d=30 \mathrm{~mm} / 7 /$. La fréquence $\nu_{1}$ est donc quel que soit le cas très superrieure à la fréquence de mesure, le module est alors complètement relaxê et

$$
\frac{\Delta E}{E}=\left(\frac{\Delta E}{E}\right)_{R}=\left(\frac{E_{S}-E}{E}\right)_{R} \quad \text { et } \quad Q^{-1}=\left(\frac{\Delta E}{E}\right)_{R} \frac{6}{\pi^{2}} \frac{f}{\nu_{1}}
$$

Une estimation de $E_{S}$ par extrapolation à $20^{\circ} \mathrm{C}$ de la partie de la courbe $E=f(T)$ pour $T>T_{C}$ nous a permis d'évaluer $\left(\frac{\Delta E}{E}\right)_{R} \simeq 0,6$. Dans le cas où $d=30 \mu \mathrm{m}$ on obtient alors $Q^{-1} \simeq 2,5 \cdot 10^{-5}$ et pour $d^{2}=300 \mu \mathrm{m} \quad Q^{-1} \simeq 2.510^{-3} / 7 /$. Cette derniēre valeur du frottement intérieur est bien du même ordre de grandeur que celle observée expérimentalement. Néanmoins si $Q^{-1}$ correspond au processus de relaxation microscopique, $i 1$ doit dépendre linéairement de la fréquence $f$; la comparaison des figures $2 a$ et $2 b$ montre que $Q^{-1}$ est loin d'être proportionnel à f. On doit alors conclure que la contribution du processus de relaxation par courants de Foucault microscopiques au frottement intérieur reste faible et il faut admettre comme Berry /10/ que le frottement intérieur à $\varepsilon \simeq 0 \mathrm{implique}$ une autre contribution indépendante de 1a fréquence et qui constituerait une sorte de fond continu magnéto-mécanique.

Enfin nous avons pu montrer /7/ qu'étant donnē la faible anisotropie d'origine structurale les processus de rotation de l'aimantation dans les domaines et de déplacement des parois doivent intervenir simultanément avec pour conséquence un effet $\Delta E$ de grande amp 1 itude.

\section{$V$ - APPLICATION A L'ETUDE DE LA RELAXATION STRUCTURALE}

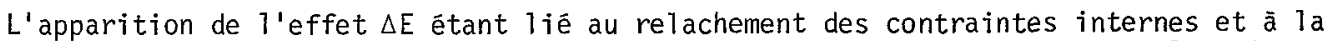
relaxation structurale du verre nous avons étudié la cinétique de cette relaxation en suivant l'évolution du module d'Young en fonction du temps pour trois températures de recuit 250,275 et $300^{\circ} \mathrm{C}$. Les vieillissements sont réalisés in situ, chaque recuit est précédé d'une montée en température à $1 \mathrm{k} / \mathrm{mn}$ et suivi d'un refroidissement rapide. Le module est mesuré à $20^{\circ} \mathrm{C}$. Les résul tats de cette étude sont représentés sur la figure 3 . Pour chaque température on peut définir un temps $t_{5}$ à partir auquel 1 a valeur du module se stabilise. Le temps $t_{S}$ suit une $10 i$ d'Arrhénius comme le montre la figure 4 . On a également reporté sur cette figure les temps to qui correspondent au ternps à partir duquel le phënomène de mise en ordre chimique devient prépondérant par rapport au phēnomène de mise en ordre topologique au cours de la relaxation structurale ; ce temps $t_{0}$ a été déterminé par ailieurs $/ 7 \%$. On constate que les droites correspondant respectivement a $t_{0}$ et $t_{s}$ sont parallēes, c'est-àdire que les ênergies d'activation apparentes sont identiques. 


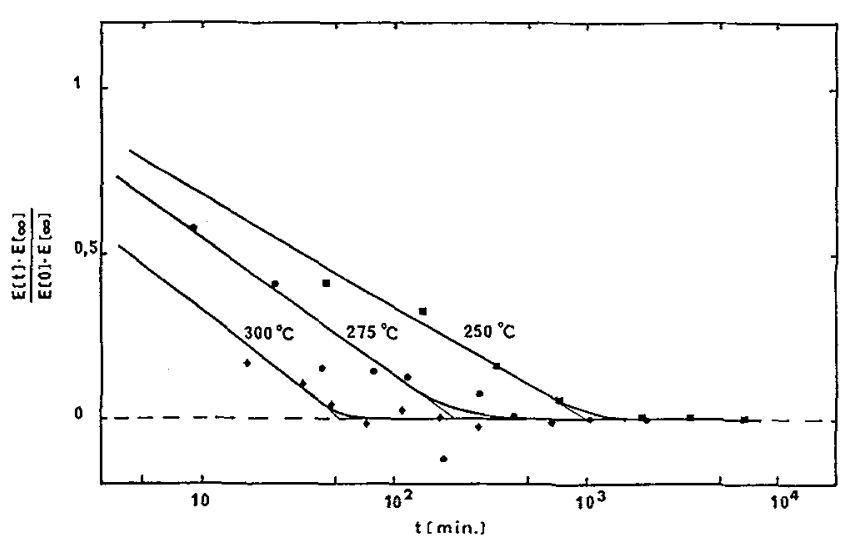

Figure 3 : Cinêtiques normalisées J'évolution du module d'Young du verre Fe83 $\mathrm{B}_{14} \mathrm{Si}_{1,5} \mathrm{C}_{1,5}$ lors de recuits isothermes $\bar{a}, 250,275$ et $300^{\circ} \mathrm{C}$.

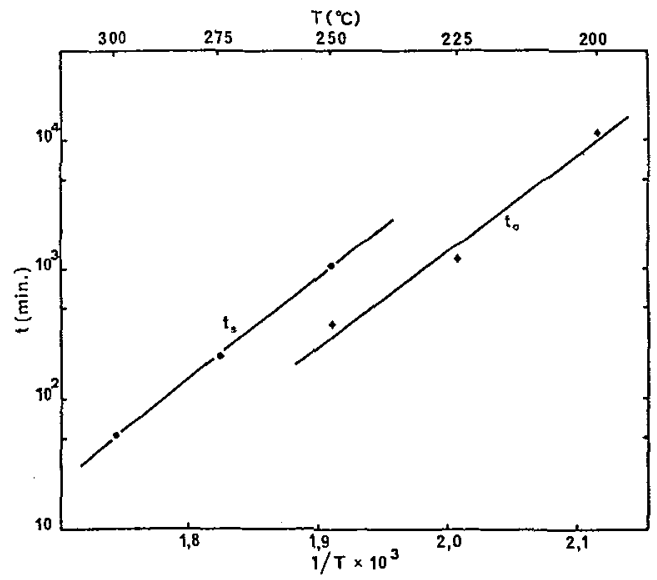

Figure 4 : Diagramme d'Arrhénius illustrant la dépendance en température de $t_{S}$ et to.

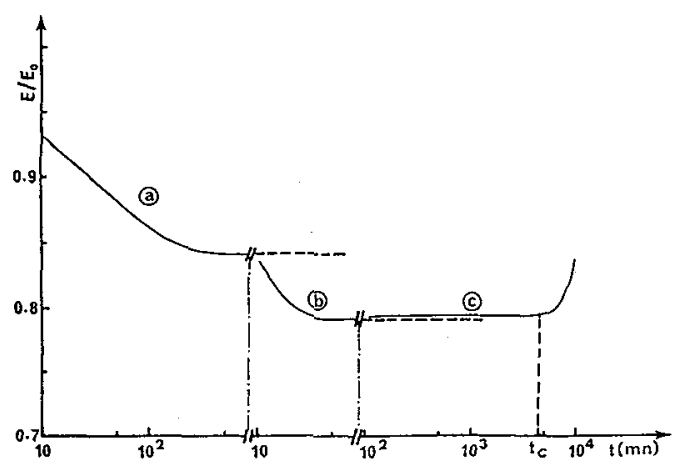

Figure 5 : Variation du module d'Young en fonction du temps de traitement pour un échantilion recuit d'abord à $275^{\circ} \mathrm{C}$ (a) puis à $300^{\circ} \mathrm{C}$ (b) et de nouveau à $275^{\circ} \mathrm{C}(\mathrm{c})$.
La diminution du module d'Young, associée à l'effet $\Delta E$ est donc essentiellement liée à la relaxation structurale topologique par l'intermédiaire des contraintes internes. Par contre, le phénomẽne de mise en ordre chimique ne semble pas affecter la valeur du module d'Young. Notons en outre que, pour un même échantillon, la valeur stabilisée du module obtenue après traitement pour différentes températures est d'autant plus faible que la température de recuit est élevée. On peut donc en conclure que 1 'effet $\Delta E$ dans le verre $\mathrm{Fe}_{83} \mathrm{~B}_{14}$ Si $1,5 \quad C_{1,5}$ "stabilisé" augmente avec la température du recuit de stabilisation, augmentation qui peut être attribuée à un niveau de contraintes internes d'autant plus bas que le recuit est réalisé à une température plus éievée.

Par ailleurs, nous avons cherchè à savoir si l'on pouvait passer réversiblement de la valeur de $E$ obtenue après stabilisation à $300^{\circ} \mathrm{C}$ à celle obtenue après stabilisation à $275^{\circ} \mathrm{C}$. Les résultats sont montrés sur la figure 5 . On constate que le recuit à $275^{\circ} \mathrm{C}$ consécutif à celui à $300^{\circ} \mathrm{C} n$ 'a pour conséquence qu' une très faible remontée du module d'Younc par rapport au niveau de stabilisation à $300^{\circ} \mathrm{C}$, la remontée rapide qui $s^{\prime}$ amorce à partir de $t_{c}$ correspond à 7 'apparition des premiers cristalitites de fer a. Il semble donc que la diminution du niveau de stabilisation du module quand on augmente 1a température de recuit est un phénomène essentiellement irréversible. 
VI - CONCLUSION

Cette étude d'un verre métallique $\mathrm{Fe}_{83} \mathrm{~B}_{14} \mathrm{Si}_{1,5} \mathrm{C}_{1,5}$ nous a permis de mettre en évidence un effet magnétoélastique de grande amplitude et un amortissement magnétomécanique fortement sensibles à l'état structural du matēriau. Leur développement, accompagnant 1a relaxation structurale topologique, est dû au relachement des contraintes internes qui dans 1 'état brut d'hypertrempe limitent le mouvement des parois et la rotation de l'aimantation des domaines. L'amorce de la cristallisation primaire de fer $\alpha$ est suffisante pour faire disparaitre 1 'effet $\Delta E$ et la plus grande partie de l'anomalie de module entre état vitreux et état cristallin.

$L$ 'analyse de 7 'effet $\Delta E$ et de 7 'amortissement magnétomécanique nous a montré que :

(i) pour les valeurs de la déformation considérées, la contribution hystérétique est faible, (ii) la relaxation par courants de Foucault ne suffit pas à expliquer le niveau de frottement intérieur à faible déformation. Il faut admettre 1 'existence d'une autre contribution, indépendante de la fréquence et constituant une sorte de fond continu magnétomécanique.

\section{Rēférences}

1. D.C. TIAN, F. FOUQUET, F. GUYOT, C. MAI, J. PEREZ, Mat. Sci Eng. 53 (1982) 179.

2. H.V. KUNZI, K. AGYEMAN, Proc. European Conf. on Internal Friction and Ultrasonic Attenuation in Solids, Manchester, (1979), 371.

3. B.S. "BERRY, in "Metallic Glasses", A.S.M., Metals Park (Ohio), (1978) 161.

4. E. TOROK, G. HAUSCH, Proc. 3 rd Int. Conf. on Rapidly quanched Metals, Brigton, vol. 2, (1978) 105.

5. T. SOSHIRODA, M. KOIWA, T. MASUMOTO, J. Non. Cryst. Solids, 22 (1976) 173.

6. S. KISS, G. POSGAY, I.Z. HARANGOZO, F.J. KEDVES, Proc. 7th Int. Conf. on Internal friction and Ultrasonic attenuation in Solids, J. Physique 42-C5 (1981) 529.

7. F. GUYOT, Thèse Docteur Ingénieur INSA LYON (1982)

8. B.S. BERRY, W.C. PRITCHET, A.I.P. Conf. Proc., New-York, 34 (1979) 292.

9. R.C. O'HANDLEY, M.C. NARASIMHAN, M.0. SULLIVAN, J. Appt. Phys. 50 (1979) 16633.

10. B.S. BERRY, W.C. PRITCHET, J. AppT. Phys. 47 (1976) 3295.

11. R. HASEgAWA, R.C. O'HANDLEY, L.I. MENDELSOHN, A. I.P. Conf. Proc., New-York, 34 (1979) 298. 